\title{
The supracerebellar-transtentorial approach to posteromedial temporal lesions in children with refractory epilepsy
}

\author{
Alexander G. Weil, MD, FRCSC, Andrew L. Middleton, MD, Toba N. Niazi, MD, John Ragheb, MD, \\ and Sanjiv Bhatia, MD
}

Division of Pediatric Neurosurgery, Department of Neurosurgery, Miller School of Medicine, University of Miami, Miami Children's Hospital, Miami, Florida

\begin{abstract}
OBJECT Operations on tumors of the posteromedial temporal (PMT) region, that is, on those arising from the posterior parahippocampal, fusiform, and lingual gyri, are challenging to perform because of the deep-seated location of these tumors between critical cisternal neurovascular structures and the adjacent temporal and occipital cortexes. Traditional surgical approaches require temporal or occipital transgression, retraction, or venous sacrifice. These approaches may result in unintended complications that should be avoided. To avoid these complications, the supracerebellar-transtentorial (SCTT) approach to this region has been used as an effective alternative treatment in adult patients. The SCTT approach uses a sitting position that offers a direct route to the posterior fusiform and lingual gyri of the temporal lobe. The authors report the feasibility, safety, and efficacy of this approach, using a modified lateral park-bench position in a small cohort of pediatric patients.
\end{abstract}

METHODS The authors carried out a retrospective case review of 5 consecutive patients undergoing a paramedian SCTT approach between 2009 and 2014 at the authors' institution.

RESULTS The SCTT approach in the park-bench position was used in 3 boys and 2 girls with a mean age of 7.8 years (range 13 months to 16 years). All patients presented with a seizure disorder related to a tumor in a PMT region involving the parahippocampal and fusiform gyri of the left $(n=3)$ or right $(n=2)$ temporal lobe. No procedure-related complications were observed. Gross-total resection and control of seizures were achieved in all cases. Tumor classes and types included 1 Grade II astrocytoma, 1 pleomorphic xanthoastrocytoma, 1 ganglioglioma, and 2 glioneural tumors. None of the tumors had recurred by the mean follow-up of 22 months (range 1-48 months). Outcomes of epileptic seizures were excellent, with seizure symptoms in all 5 patients scoring in Engel Class IA.

CONCLUSIONS The SCTT approach represents a viable option when resecting tumors in this region, providing a reasonable working corridor and low morbidity. The authors' experience in a cohort of pediatric patients demonstrates that complete resection of the lesions in this location is feasible and is safe when involving an approach that involves using a park-bench lateral positioning.

http://thejns.org/doi/abs/10.3171/2014.10.PEDS14162

KEY WORDS posteromedial temporal; fusiform gyrus; supracerebellar transtentorial; parahippocampal gyrus; refractory epilepsy; hippocampus; park-bench position; oncology; technique

$\mathrm{L}$ ESIONS involving the middle and/or posteromedial temporal (PMT) regions and arising in the parahippocampal, fusiform, and anterior lingual gyri are challenging to treat because they are located in deep-seated regions, proximal to critical neurovascular structures, and are therefore difficult to access via surgical approaches. ${ }^{1,3}$ Conventional approaches to resect these tumors include the transtemporal-transventricular, subtemporal, supratentori- al-infraoccipital, occipital-transtentorial, interhemisphericparietooccipital, and pterional-transylvian routes. ${ }^{1-3,9,15,19,21}$ Exposure of the PMT region through these traditional approaches increases the risk of eloquent visual, language, and limbic temporal lobe pathway damage due to vascular injury or tissue transgression or retraction. . $^{3,9,19,21,24}$

The subtemporal approach offers access to the PMT region at the expense of retraction of the temporal lobe.

ABBREVIATIONS ECOG = electrocorticography; PHG = parahippocampal gyrus; $\mathrm{PMT}=$ posteromedial temporal; $\mathrm{SCTT}=$ supracerebellar-transtentorial. SUBMITTED April 1, 2014. ACCEPTED October 6, 2014.

INCLUDE WHEN CITING Published online November 14, 2014; DOI: 10.3171/2014.10.PEDS14162.

DISCLOSURE The authors report no conflict of interest concerning the materials or methods used in this study or the findings specified in this paper. 
Because of the inclination of the tentorium, temporal lobe retraction increases with a more posterior location of the lesion. ${ }^{1}$ Besides the possibility of parenchymal retraction injury, the vein of Labbé is also particularly vulnerable to injury in this approach. ${ }^{1}$ The transtemporal-transventricular approach necessitates dissection through normal tissue of the temporal lobe and the temporal horn. ${ }^{1}$ Again, the more posterior the lesion, the higher the risk of injury to the optic radiations and eloquent speech centers of the dominant hemisphere. This approach is especially contraindicated for lesions of the dominant hemisphere. Pterional-transsylvian and interhemispheric-parietooccipital approaches are associated with limited exposure to the more posterior part of the mesial temporal lobe and require significant brain retraction. ${ }^{3,21}$

The supracerebellar-transtentorial (SCTT) approach has been described as a safe alternative to resect lesions in the PMT region in adults, but only 4 uses of this method have been previously reported on in pediatric patients. ${ }^{3,9,13,24,28}$ The SCTT approach offers an alternative route to the PMT region, while avoiding the risks of injury to the superficial venous drainage of the temporal lobe and of injuries associated with temporal lobe retraction or with transgression through normal brain tissue. Thus, the SCTT approach may reduce the risk of producing surgery-related neurological deficits.

Here, we report the feasibility, safety, and efficacy of the SCTT approach for resecting tumors affecting the middle and PMT brain regions in patients who have epilepsy refractory to pharmacotherapy. Furthermore, whereas all previous cases of the SCTT approach have been performed with the patient being in the sitting position, we describe a variation of the SCTT approach through a lateral park-bench position and discuss the rationale for and advantages of this approach.

\section{Methods}

After approval by the institutional review board, we performed a retrospective review of medical records and imaging studies of all patients who underwent an SCTT approach at Miami Children's Hospital. We identified 5 patients who underwent this approach for resection of tumors at the Miami Children's Hospital between 2009 and 2014.

\section{Preoperative Assessment and Patient Selection}

All patients exhibited medically refractory epilepsy and underwent a standard detailed noninvasive preoperative epilepsy workup. The indications for surgery were the following: 1) to treat refractory seizures in patients whose lesions were localized to the posteromedial temporal region, 2) to obtain a histopathological diagnosis of the PMT lesion, and 3) to achieve tumor control.

\section{Surgical Technique}

All procedures were performed under general anesthesia. Indwelling Foley catheters, arterial lines, and peripheral intravenous lines were placed in all cases.

The patients were positioned in the three-quarter lateral park-bench position (Fig. 1). The patient's head was placed in a Mayfield clamp and flexed and rotated contralaterally, thus creating a direct access to the tentorium that enabled the SCTT approach to the medial posterior temporal lobe (Fig. 2A). The patient's body was supported by pillows and strapped securely to the operating table. A combination of a headrest and a Mayfield 3-point fixation (Patient 1) or a Mayfield 3-point fixation clamp alone (Patients 2-4 and 5) were used to immobilize the skull.

Stereotactic neuronavigation was used in Patients 1-3 and 5; technical limitations of the navigation system precluded its use in Patient 4.

An incision was planned in the suboccipital region, and a linear strip of hair was shaved in a paramedian fashion ipsilateral to the involved temporal lobe (Fig. 2A). After infiltration of local anesthesia with epinephrine, a vertical linear incision was made such that it extended above the superior nuchal line (above the transverse sinus) and down to the base of the occiput. The muscle and soft tissues were dissected and held open with self-retaining retractors. The craniotomy was performed such that it extended just above the transverse sinus, abutting the superior sagittal sinus and extending laterally to near the transverse-sigmoid junction. About two-thirds of the craniotomy extended to the suboccipital region but did not involve the foramen magnum (Fig. 2B). The craniotomy was carried out to nearly the sigmoid sinus on the side of interest and across the midline on the contralateral side. This facilitated a more medial access and enabled removal of the lateral extents of the tumor.

The dura was opened in a V-shaped manner; the opening was based superomedially, just inferior to the torcula, sometimes extending to the contralateral side across the inferior sagittal sinus and the lateral border extending to the limit of the cranial opening. Both cranial and dural openings were made as wide as possible, to create a good working corridor and help avoid sacrifice of the bridging vein. The dural opening was limited superiorly by the transverse sinus, which was exposed to facilitate superior retraction of the dural flap (Fig. 2C). After opening of the dura, the occipital surface of the cerebellum was carefully retracted in the midline to drain CSF from the cisterna magna. This drainage significantly relaxed the cerebellum.

The bridging veins between the tentorial surfaces of the cerebellum and tentorium were coagulated and divided to further relax the cerebellum (Fig. 2C). The tentorium was coagulated and incised laterally to the straight sinus in a radial manner, from lateral to medial (Fig. 2D). Bleeding edges were coagulated and the durotomy carried to the tentorial incisura by progressively coagulating the dural leaves. The tentorial incision allowed placement of a retractor above the tentorium behind its petrous attachment. In addition, large tentorial lakes may be present, especially in very young children, and these were avoided whenever possible. Preoperative venous-phase MR angiography may be helpful in identifying large venous sinuses within the tentorium, and image guidance may be used to help avoid these sinuses during the operation. Importantly, the vein of Rosenthal may drain into the tentorium, and this should be considered a contraindication to the SCTT approach..$^{21}$ Care should also be taken to avoid sacrificing the paramedian tentorial bridging veins whenever possible. The midline tentorial bridging veins and the petrosal vein (laterally) 




FIG. 1. Medical illustration showing the SCTT approach to a tumor involving the middle temporobasal and PMT regions. Copyright Alexander G. Weil. Published with permission.

must be preserved because they represent the predominant venous drainage of the posterior fossa. ${ }^{21}$ Temporary arrhythmia or bradycardia, mediated through the trigeminalvagal pathway, has been reported during this step of tentorial manipulation; therefore, at this stage, communication with the anesthesiologist is important. ${ }^{28}$

The undersurface of the temporal lobe was visualized, and a self-retaining retractor was then placed through the tentorial opening into the supratentorial space to depress the tentorium in order to create a larger working corridor.
The quadrigeminal and posterior ambient cisterns were then exposed; this typically allowed for visualization of the straight sinus and vein of Galen medially and of the basal vein of Rosenthal. Opening of the thick arachnoid of the quadrigeminal and posterior ambient (perimesencephalic) cisterns allowed for visualization of the posterior cerebral arteries. The trochlear nerve should first be identified as it exits the posterior midbrain below the inferior colliculus and should be followed anteriorly as it travels the quadrigeminal and ambient cisterns until it tucks un- 

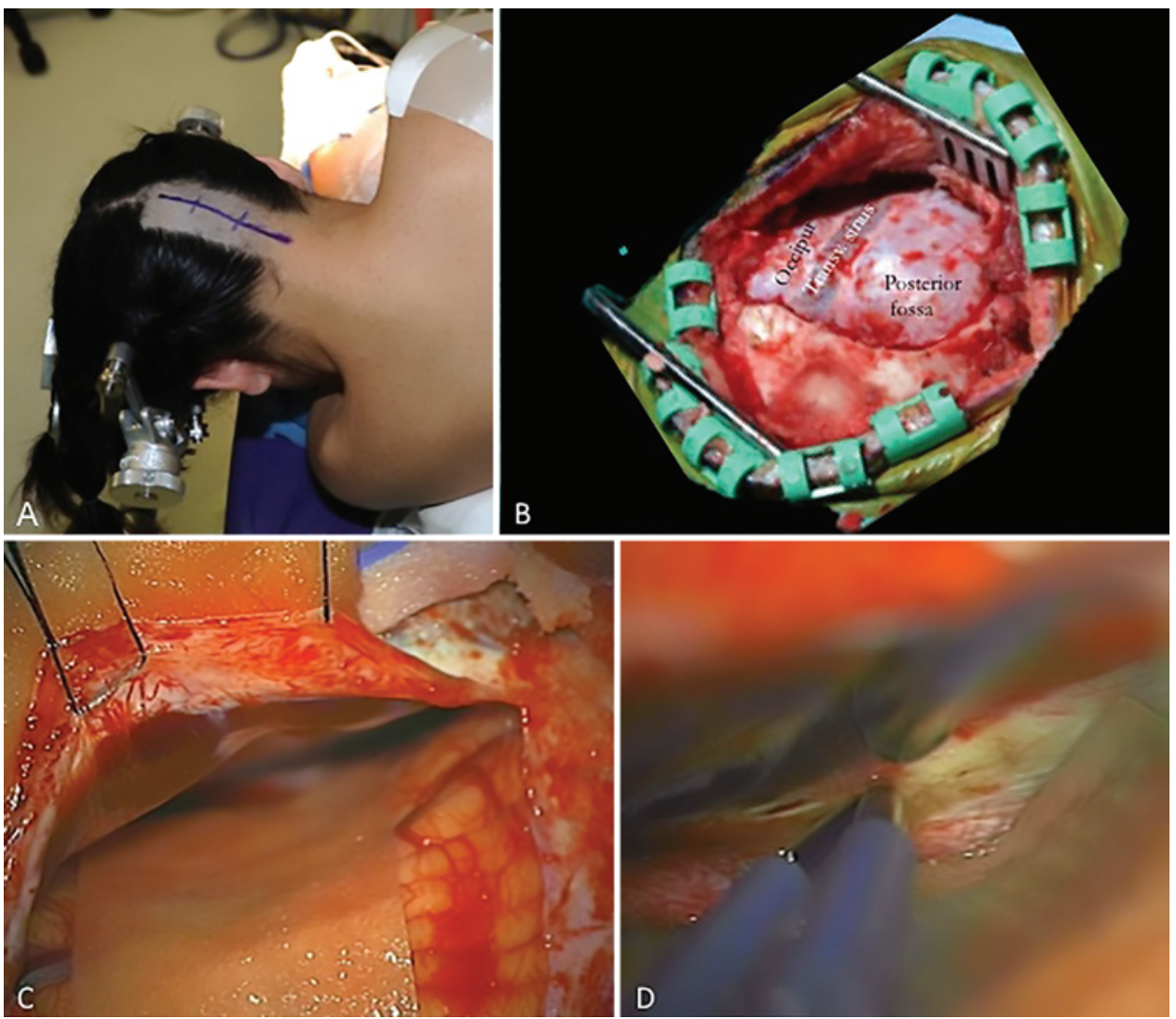

FIG. 2. Surgical technique of the SCTT approach. A: The patient is placed in the lateral park-bench position. B: The craniotomy exposes one-third above and two-thirds below the transverse (transv) sinus. C: A V-shaped durotomy, superior tenting of the supratentorial dura, selective sacrifice of the cerebellar bridging veins, and cerebellar relaxation have been performed. D: Tentorial coagulation and incision is carried out in a medial to lateral direction, avoiding incursion to the tentorial vein and the trochlear nerve.

der and pierces the tentorium. The nerve extends below the tentorium before entering the dural canal, usually at the anteroposterior midpoint of midbrain (for example, at the posterior border of the cerebral peduncle). Thus, the safest location to incise the tentorium is posterior to the tectum of the midbrain..$^{20}$ As such, injury to the trochlear nerve is never a major risk. Frameless image guidance can greatly aid at this stage.

With the tentorium opened and retracted, the undersurface of the PMT lobe was visualized. In our series, the tumor typically obscured the normal anatomy of the fusiform and parahippocampal gyri, allowing direct visualization of the tumor and of the infiltrated cortex. In cases where refractory epilepsy is the primary presentation, it is possible to place a subdural strip under the parahippocampal gyrus to perform electrocorticography (ECoG), which was done in 2 of the cases. One patient showed 2 pathological changes, one of which was an area of cortical dysplasia in the adjacent cortex, and it was unclear whether the seizures were arising from the area around the tumor or the region of cortical dysplasia. In 3 of the 5 cases, the hippocampus was preserved (Patients 1 and 4). In the other 2 cases, the hippocampus was either partially resected (Patient 1) because of tumor infiltration or transected (Patient 3) because of intraoperative ECoG measurements suggesting interictal epileptic activity. In this case of transection, the hippocampus was disconnected at the trigone by dividing the tail and fornix (including the fimbria).

As intratumoral decompression was performed, care was taken to respect the pial plane, which was sought medially, so as to avoid encountering the midbrain and vascular structures medially. Depending on tumor extent, the temporal horn of the ventricle was frequently entered ( $\mathrm{Pa}-$ tients 1-3 and 5). Tumor removal was carried out with a combination of suction, bipolar coagulation, and ultrasonic aspiration. The limits of the resection were easily visualized with this approach, but this delineation required frequent repositioning of the operating microscope and the operating table for adequate resection in all cases of this series. After adequate tumor resection had been achieved and hemostasis had been secured, the tentorial retractor was removed and the cavity filled with irrigating fluid to remove as much air as possible. The tentorial edges were not reapproximated, the convexity dura was closed, and the bone replaced.

\section{Postoperative Management and Outcome Assessment}

Patients were extubated immediately after surgery and remained in the intensive care unit for 24-48 hours. Postoperative brain MRI was performed in all cases to examine the extent of tumor resection. The patients were followed up, and postoperative epilepsy outcomes were assessed with the Engel classification scale. ${ }^{5}$ 


\section{Results}

\section{Patient Cohort}

The SCTT approach was used in 5 patients to treat lowgrade tumors and cortical dysplasia located in the middle and posterior parts of the mesial temporal lobe. The relevant clinical details of the patients are summarized in Table 1 . The patients were 3 boys and 2 girls, ranging in age from 13 months to 16 years (mean 7.8 years). All 5 children had a history of seizures on presentation and had a newly diagnosed tumor in the PMT region. All of the tumors involved the posterior and/or mediobasal temporal lobes, including the parahippocampal, fusiform, and adjacent anterior lingual gyri, including 3 in the left and 2 in the right hemispheres (Fig. 3).

\section{Tumor Characteristics}

The tumors in this series were one WHO Grade II astrocytoma, one WHO Grade II pleomorphic xanthoastrocytoma, one WHO Grade I ganglioglioma, and two WHO Grade II glioneural tumors. The glioneural tumors comprised a glioneuroma superimposed in the setting of an oligodendroglioma and an oligodendroglioma (without a 1p19q codeletion) within a ganglioglioma.

\section{Outcomes}

Gross-total resection was achieved in all cases as determined by postoperative MRI examination. No procedure-related complications were observed, and none of the tumors had recurred within a mean follow-up period of 22 months (range 1-48 months). Epilepsy outcomes were excellent, with all patients being free of seizures (that is, symptoms classified as Engel Class IA) at the last followup. No new memory deficits were identified postoperatively in any of the patients.

\section{Illustrative Case}

A 16-year-old girl was referred from the emergency department with a history of 2 months of recurrent seizures. An MRI scan of the brain revealed a heterogeneously enhancing mass, hypointense on T1- and hyperintense on T2-weighted images, centered in the right parahippocampal and fusiform gyri (Fig. 4 upper). A regimen of antiepileptic medicines failed to decrease seizure symptoms, and over a period of 1 month, seizure activity significantly increased. Besides the seizures, the patient had no history of other significant symptoms or diseases.

\section{Operation}

The patient underwent a right paramedian supracerebellar-transtentorial resection of the tumor without complications. Final histopathological analysis revealed a pleomorphic xanthoastrocytoma, WHO Grade II.

\section{Postoperative Course}

At follow-up 7 months postoperatively, the patient was seizure free (that is, the outcome was classified as Engel IA), and postoperative imaging indicated a gross radiological resection of the mass with no recurrence (Fig. 4 lower).

\section{Discussion}

We report a series of 5 children, 4 of whom had epilepsy refractory to pharmacotherapy and low-grade developmental tumors affecting the middle and PMT lobe. The challenges of surgical access to these tumors were compounded by the presence of associated cortical dysplasia in some of the patients, raising uncertainty about the exact location of the epileptogenic zone. The goal of this report was to demonstrate the feasibility, safety, and efficacy of accessing the middle and posteromedial regions of the temporal lobe through the suboccipital SCTT approach in children.

Safe and effective resection of tumors involving the PMT lobe through an SCTT approach begins with a thorough knowledge of the anatomy of the mediobasal temporal lobe..$^{3,21}$ This lobe is divided into anterior, middle, and posterior thirds (Fig. 5). The mediobasal temporal lobe essentially consists of temporal structures that are located medial to the collateral and rhinal sulci. The anterior third contains the uncus, amygdala, head of hippocampus, and the anterior portion of the parahippocampal gyrus (PHG). It is limited from the fusiform (that is, occipitotemporal) gyrus by the rhinal sulcus and is bordered medially by the anterior two-thirds of the cerebral peduncle and crural cistern. The anterior third is separated from the middle third by the posterior limit of the uncus. The middle third of the mediobasal temporal lobe is separated from the posterior third by the anterior splenial line (a vertical line at the anterior border of the splenium of the corpus callosum, which is also located behind the collicular midbrain). This middle third of the mediobasal temporal lobe has an inferior portion composed of the PHG and a medial portion composed of the PHG, subiculum, dentate gyrus, fimbria of fornix, and body of the hippocampus. It is limited laterally by the collateral sulcus, and its medial border lies

TABLE 1. Characteristics of the patients in this study*

\begin{tabular}{|c|c|c|c|c|c|}
\hline $\begin{array}{l}\text { Patient } \\
\text { No. }\end{array}$ & Age, Sex & $\begin{array}{l}\text { Presentation \& Length of } \\
\text { Symptomatic Period }\end{array}$ & Tumor Location & Tumor Type & $\mathrm{FU}$ (mos) \\
\hline 1 & $13 \mathrm{mos}, \mathrm{M}$ & CPS, 3 mos & Lt PHG & WHO Grade II diffuse astrocytoma & 48 \\
\hline 3 & $7 \mathrm{yrs}, \mathrm{F}$ & CPS, 6 yrs; RMP & Lt PHG \& FG & WHO Grade II oligodendroglioma in ganglioglioma & 7 \\
\hline 4 & 16 yrs, $\mathrm{F}$ & CPS, 2 mos & Rt PHG \& FG & WHO Grade II PXA & 7 \\
\hline 5 & $9 \mathrm{yrs}, \mathrm{M}$ & CPS, 8 mos & Lt PHG \& FG & WHO Grade I ganglioglioma & 1 \\
\hline
\end{tabular}

CPS = complex partial seizures; FG = fusiform gyrus; FU = follow-up; PXA = pleomorphic xanthoastrocytoma; RMP = radiographic mass progression.

* None of the patients in this study had a neurological deficit after surgery, all operations resulted in gross-total resections, and all patients' postoperative epilepsy was

Engel Class IA. 

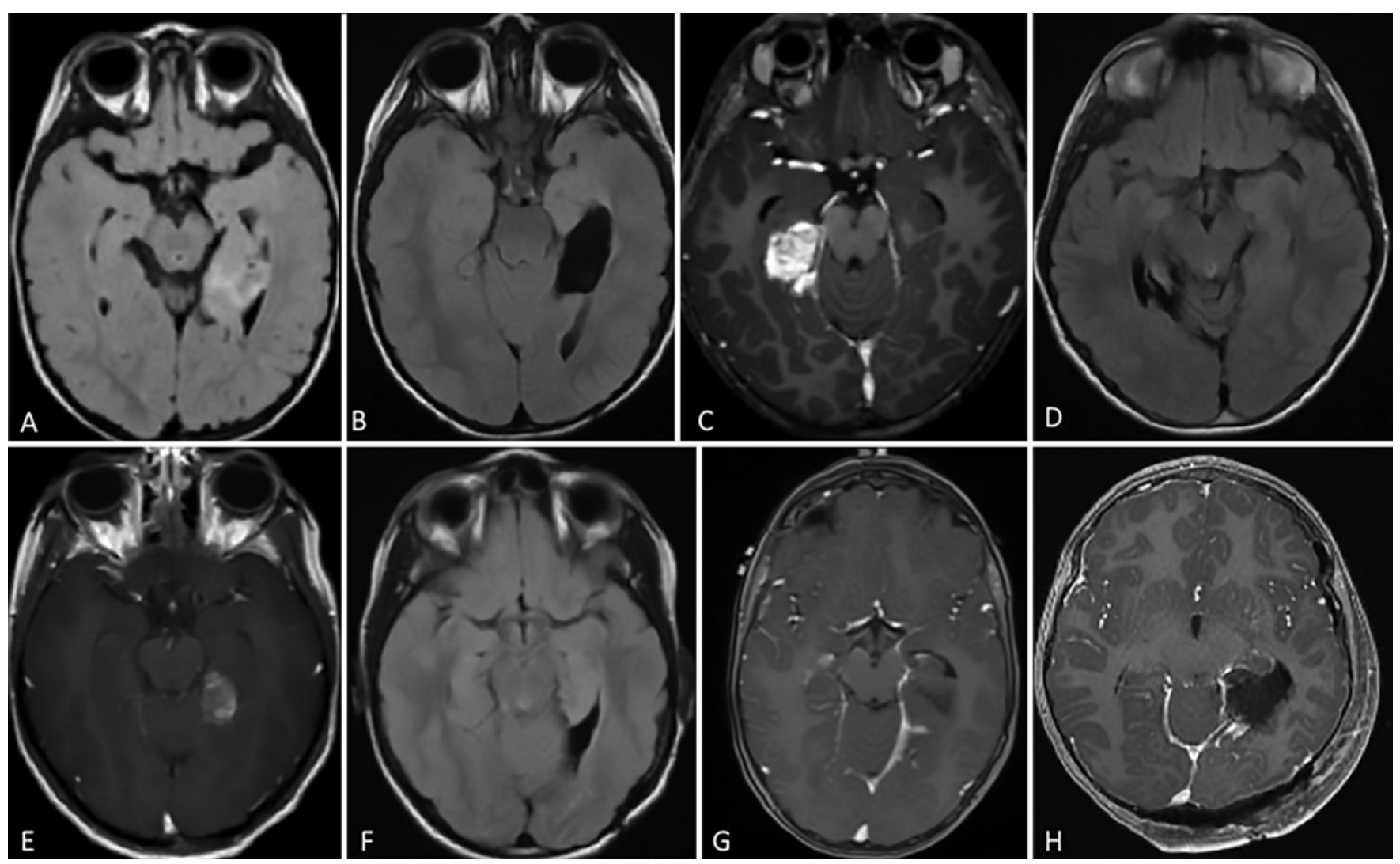

FIG. 3. Preoperative and postoperative MR images of selected cases. Preoperative (A) and postoperative (B) images of Case 1 , preoperative (C) and postoperative (D) images of Case 2, preoperative $(E)$ and postoperative $(F)$ images of Case 3, and preoperative $(\mathbf{G})$ and postoperative $(\mathbf{H})$ images of Case 5.

adjacent to the posterior third of the cerebral peduncle, midbrain tegmentum, and ambient cistern.

The posteromedial region (that is, the posterior third) consists of the posterior end of the PHG, which is divided by the anterior calcarine sulcus into the isthmus of the cingulum superiorly and the lingula of the cuneus inferiorly. This posterior mediobasal temporal lobe is limited medially by the posterior ambient and quadrigeminal cisterns and laterally by the collateral sulcus, which separates the inferior PMT region (containing the PHG and lingula) from the fusiform gyrus (Fig. 5). The posterior limit of the PMT region consists of a virtual parietotemporal line connecting the parietooccipital sulcus and preoccipital notch. ${ }^{3,4,6,10,14,21,27}$ The PMT region is in close proximity to the mesencephalic segment of the basal vein of Rosenthal as it courses in the quadrigeminal cistern and makes its entry into the vein of Galen as well as into the $\mathrm{P}_{3}$ and $\mathrm{P}_{4}$ segments of the posterior cerebral artery and its tributaries in the PMT region. This region is draped by the occipitotemporal neocortex, containing the temporal optic radiations, language pathways, and important associative areas. ${ }^{14}$ Thus, the optic radiations and the vein of Labbé limit surgical access to this region from the lateral side.

Since its initial description by Voigt and Yaşargil in $1976,{ }^{24}$ the SCTT approach has been used to access atrial tumors, ${ }^{8,11}$ petroclival or tentorial meningiomas, ${ }^{22,25}$ middle fossa lesions, ${ }^{7}$ and thalamic tumors. ${ }^{16}$ In total, 44 cases of PMT-region lesions, including 21 tumors, have been resected through an SCTT approach. ${ }^{3,13,21,24,28}$ Of these, only 4 have been performed in children. We report our results with the SCTT approach in 5 pediatric patients, all of whom were operated in the lateral park-bench position and in whom no procedure-related complications were observed. Our results compared favorably to the previous 44 cases of PMT lesions resected with an SCTT approach. Among these 44 cases, no procedure-related complications were reported. Only 1 new-onset neurological deficit has been reported in the literature: a visual deficit in a patient who underwent SCTT resection of a medial temporal tumor to treat epilepsy (Table 2), ${ }^{3,21,28}$ In addition to being safe and feasible, the SCTT approach was effective in all of our patients: a gross-total resection was accomplished in all 5 cases, and excellent control of seizures was achieved.

The rationale for using an SCTT approach to resect tumors in the PMT region in children resides in the facility of this approach to provide a good window to the PMT region, while avoiding damage to the temporal lobe and its eloquent pathways. Cadaveric studies have shown that the SCTT approach helps reach the entire length of the medial temporal region, showing the utility of this approach to treat tumors that extend beyond the PMT region into the temporal pole. ${ }^{3,21}$ By avoiding lateral transgression into the temporal brain regions, this approach may avoid creating new cortical injuries and gliosis arising from transtemporal approach-related cortical damage, and may thus reduce the risk for postoperative recurrence of seizures. ${ }^{18}$ 

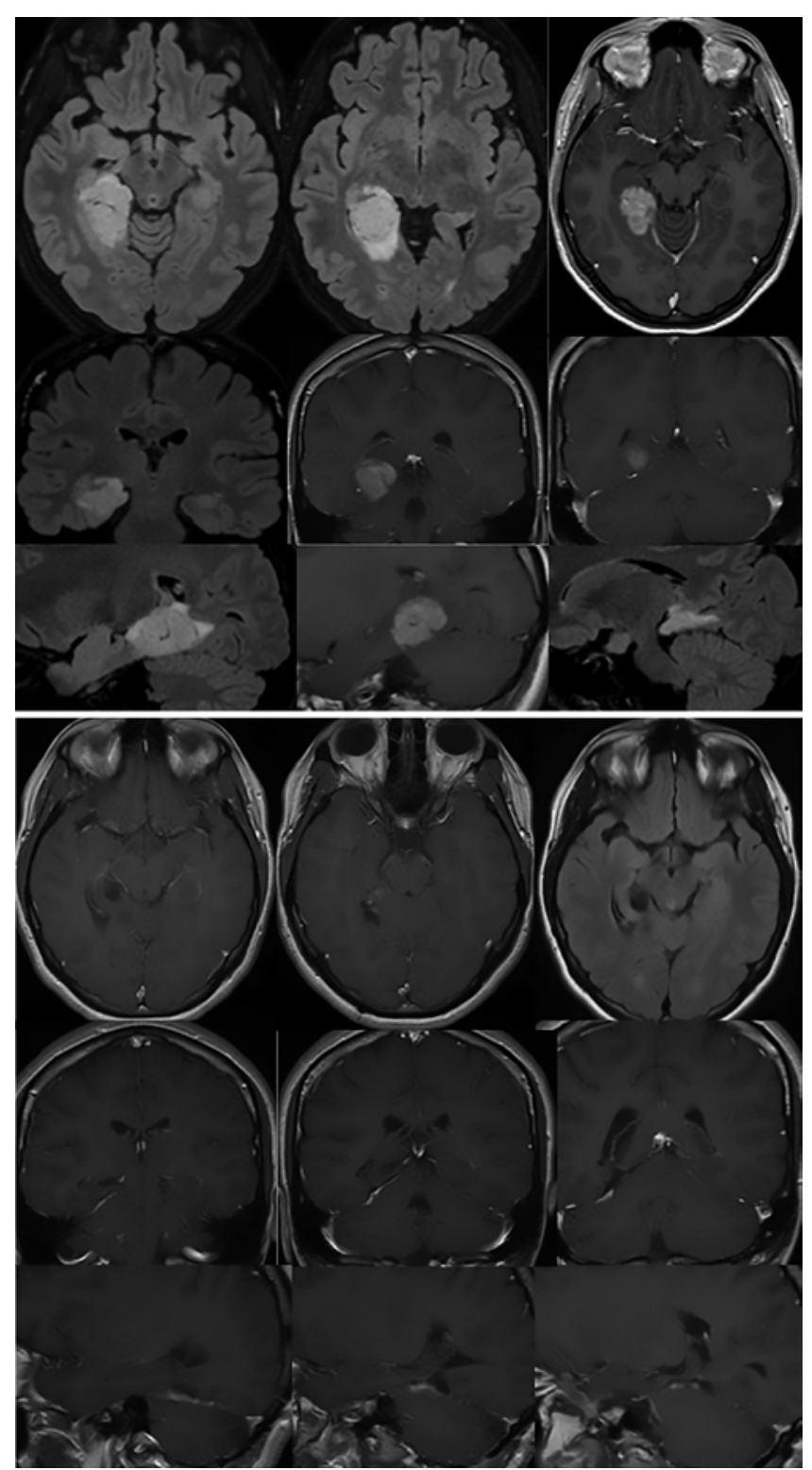

FIG. 4. Case 4. Preoperative and postoperative MRI series. Upper: Preoperative axial, coronal, and sagittal MRI scans showing a heterogeneously enhancing mass, hypointense on T1- and hyperintense on T2weighted images, centered in the right parahippocampal and fusiform gyri. Lower: Postoperative axial, coronal, and sagittal MRI scans showing gross-total resection in the same patient.

There are clear advantages of the SCTT approach over the more traditional approaches to the PMT region, including anterior approaches such as the pterional-transylvian approach; lateral approaches such as a standard subtemporal, supratentorial-infraoccipital, or transcortical (that is, temporal) approach; and posterior approaches such as an occipital-transtentorial or a parietooccipital interhemispheric approach. ${ }^{9,15,19,21,26}$

Lateral approaches, including the transtemporal and lateral subtemporal approaches, offer good access to the medial temporal region, but have some drawbacks when used for more posteriorly located medial temporal lesions. ${ }^{15}$ The transtemporal approach requires transgression through normal tissues of the temporal lobe and temporal horn. ${ }^{1}$ This approach is more suited for lesions located in the anterior or middle medial temporal regions, because creation of a more posterior transcortical corridor is associated with a higher risk of injury to the optic radiations and speech centers of the dominant hemisphere than more anterior approaches. ${ }^{1}$ The subtemporal approach avoids neocortical transgression and injury to the optic radiations ${ }^{19,20}$ However, this approach is associated with retraction of the temporal lobe and with a risk of vein of Labbé sacrifice. A more posterior-oriented supratentorial-infraoccipital variation of the subtemporal approach has been described, which is performed to effectively approach and resect epileptogenic lesions in PMT regions. ${ }^{17,19}$ However, as the standard subtemporal approach, it may be associated with significant temporooccipital lobe retraction.

Traditional posterior approaches to lesions in PMT regions have several shortcomings. ${ }^{3,9}$ The interhemisphericparietooccipital approach allows only limited exposure of the more lateral PMT regions, and the access provided by this approach is restricted to the posterior portion of the mediobasal temporal lobe. Also, retraction of the occipital lobe may result in new-onset visual deficits. ${ }^{3}$

Finally, a long, narrow working corridor through the basal cisterns limits more anterior approaches, such as the pterional-transsylvian approach. Although this approach may reach the anterior and middle medial temporal regions, it may not extend far enough to reach the posterior part of the mesial temporal lobe..$^{3,20,24,28}$ Use of the SCTT approach to resect lesions in the medial temporal region that extend along the length of the medial temporal lobe into the PMT regions typically requires a second approach (for example, an interhemispheric, parietooccipital approach) to resect residual lesions in the PMT regions..$^{21,24}$ The reported disadvantages and complications associated with these various approaches motivated us to resect epileptic lesions in the PMT regions with the SCTT approach.

The tenets and the technique for the SCTT approach have been described in great detail previously. ${ }^{3,9,21,24,28}$ All previous SCTT approaches have been performed with patients in the sitting or semisitting position, with the tentorium parallel to the floor. ${ }^{3}$ Here, we report a variation of this approach that involves placing the patient in a lateral park-bench position. The advantage of this position is that it helps avoid the potential complications associated with a sitting-position operation, including formation of air embolisms..$^{12,21}$ The park-bench position also offers an alternative position for patients with right-to-left cardiac shunts who cannot undergo operations while in a sitting position. We would like to emphasize the following steps: during positioning, it is important to obtain good contralateral head rotation (that is, rotating the nose toward the floor). This brings the ipsilateral tentorium to a plane perpendicular to the approach, giving the surgeon a direct line of sight to the PMT region (Figs. 1 and 2).

In children, the tentorium may contain significantly more venous lakes than in adults. Therefore, while incising the tentorium, the surgeon should be attentive to this feature specific to pediatric patients. A detailed knowledge of the medial temporal anatomy and adjacent structures is critical to safely perform this approach. ${ }^{6,27}$ When perform- 

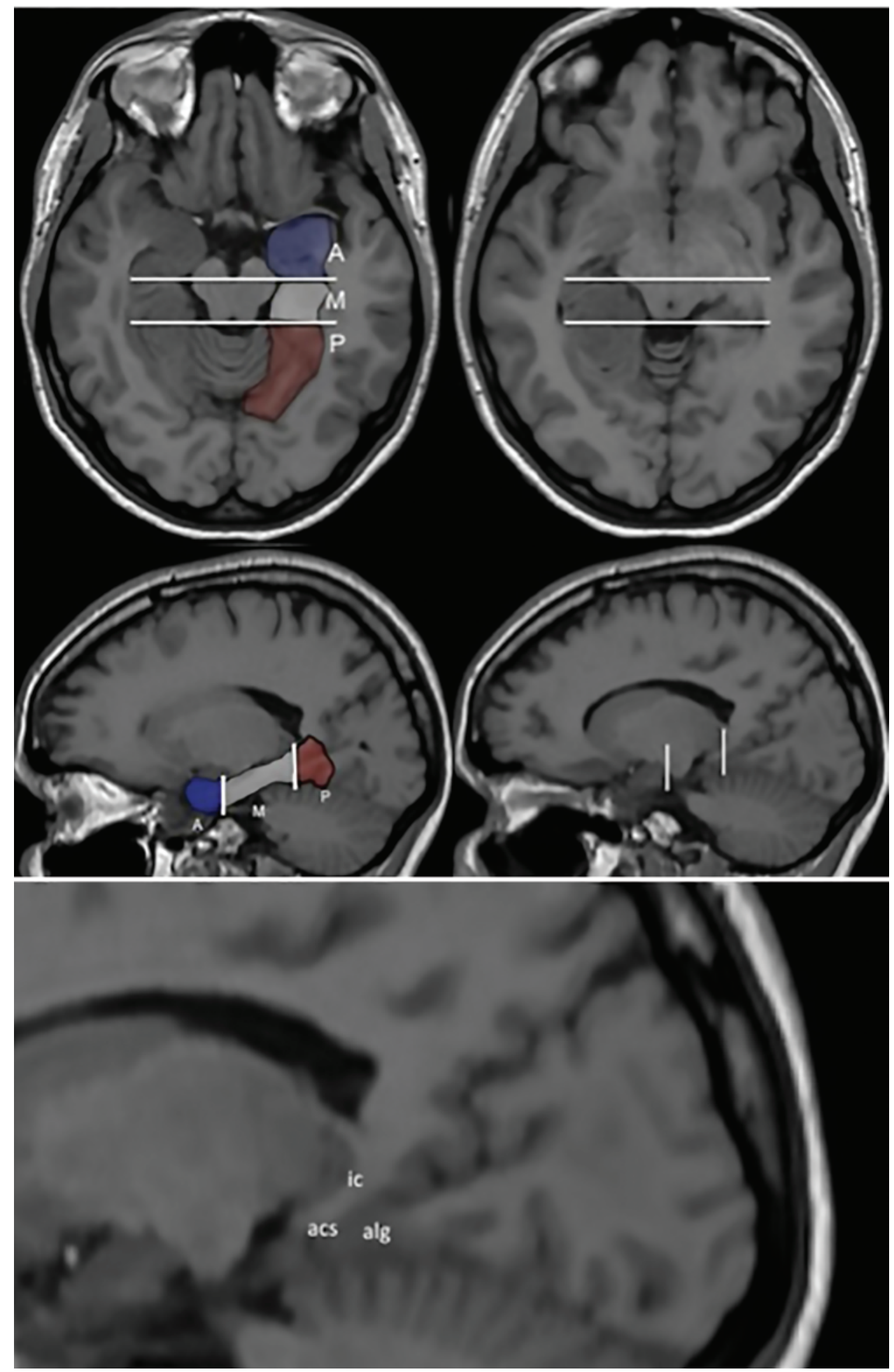

FIG. 5. Anatomy of the mediobasal temporal lobe. Upper: Axial T1-weighted and sagittal T2-weighted images indicate the division of the mediobasal temporal lobe into anterior ( $\mathrm{A}$, shaded in blue), middle $(\mathrm{M}$, shown in white), and posterior ( $\mathrm{P}$, shaded in red) portions. The anterior portion is separated from the middle portion by a transverse line along the posterior border of the uncus. The middle portion is separated from the posterior mediobasal temporal lobe by the anterior splenial line, a vertical line at the anterior border of the splenium (at the tectum colliculi of the midbrain). Lower: The posterior mediobasal temporal lobe consists of the posterior PHG, which is divided by the anterior calcarine sulcus (acs) into the isthmus of cingulum (ic) and the anterior lingual gyrus (alg). 
TABLE 2. Summary of a literature review of the use of the SCTT approach for resecting lesions in PMT regions*

\begin{tabular}{lrll}
\hline \multicolumn{1}{c}{ Authors \& Year } & $\begin{array}{c}\text { No. of } \\
\text { Patients }\end{array}$ & $\begin{array}{c}\text { Position } \\
\text { During Op }\end{array}$ & New-Onset Deficit \\
\hline Yonekawa et al., 2001 & 16 & sitting & none \\
\hline Moftakhar et al., 2008 & 1 & sitting & none \\
\hline Türe et al., 2012 & 15 & sitting & none \\
\hline de Oliveria et al., 2012 & 12 & semisitting & $1 / 12$ new visual deficit \\
\hline Present study & 5 & park bench & none \\
\hline
\end{tabular}

* None of the patients in any of the studies experienced any complications.

ing tumor resection, preserving the pial plane medially is important to avoid transgressing the midbrain or encountering the posterior cerebral artery or superior cerebellar artery. When epilepsy refractory to pharmacotherapy is the principal presentation, intraoperative ECoG may help surgeons make decisions about the extent of the tissue resection.

The SCTT approach may be used to access the entire length of the mediobasal temporal region. ${ }^{3,21}$ However, access to the anterior structures, such as the amygdala and the uncus, can be challenging. ${ }^{3,21}$ These anterior structures are more difficult to expose as they extend anteromedially, wrapping around the anterior midbrain adjacent to the crural cistern. In addition, the surgeon's view may be obstructed by the petrous ridge. ${ }^{21}$ Resection of these structures is feasible, especially in cases where they have been infiltrated or replaced with tumor, in which case internal debulking and resection is carried forward anteriorly. In addition to using an endoscope and curved suction, some authors have reported using cotton balls placed below the PHG to bring the anterior part into view. ${ }^{21}$ In 1 of our cases, the ECoG suggested persistent epileptic activity from anterior mesial structures, and the hippocampus was disconnected posteriorly at the level of the fimbria.

The paramedian SCTT approach enables only limited exposure of more lateral temporal neocortical structures (for example, the inferior temporal gyrus), especially more anteriorly. The anterior PHG is hidden by the petrous ridge. ${ }^{21}$ The fusiform gyrus may be resected posteriorly; however, this approach becomes more difficult as the resection extends anteriorly. More lateral structures (for example, the inferior temporal gyrus) cannot be reached unless the tumor extends into these structures laterally. Therefore, the SCTT approach should be reserved for epileptogenic lesions limited to structures including and medial to the fusiform gyrus. We did not encounter ECoG activity originating from the lateral inferobasal temporal lobe (that is, from the inferior temporal gyrus). Had we observed such activity after the initial SCTT resection, we would have had to consider approaching this region through a second lateral approach.

The potential disadvantages of the SCTT approach may include the risk of cerebellar venous infarction or of hemorrhage resulting from sacrifice of the bridging veins during the exposure. ${ }^{21,23}$ In our series, as in all reported cases of SCTT approaches, $3,7-9,11,16,21,22,24,25,28$ no CSF leakages were observed. Furthermore, the paramedian approach may avoid the median vermian bridging veins, thus avoiding venous sacrifice in three-quarters of patients. ${ }^{21}$

The SCTT approach brings the transverse sinus and medial venous structures (such as the basal vein of Rosenthal) into surgical areas, putting them at increased risk for injury. This approach also has a significant learning curve. Frameless image guidance was used in the first 3 and in the last case. It was found to be very helpful and reliable for planning the tentorial opening and for assessing the anterior and lateral extent of the lesionectomy. However, as the surgeon becomes familiar with the anatomy of this region, neuronavigation ceases to be of vital importance.

\section{Conclusions}

Tumors arising from the parahippocampal and fusiform gyri of the PMT lobe require resections that present technical challenges for the neurosurgeon. Various approaches to this region have been described, but many of these require significant retraction or transgression into healthy tissues to accomplish successful resection. The paramedian SCTT approach represents a viable approach to tumors of this region with low morbidity and a reasonable working corridor. Our experience with 5 patients demonstrates the safety and viability of this approach in a pediatric cohort.

\section{References}

1. Campero A, Tróccoli G, Martins C, Fernandez-Miranda JC, Yasuda A, Rhoton AL Jr: Microsurgical approaches to the medial temporal region: an anatomical study. Neurosurgery 59 (4 Suppl 2):ONS279-ONS308, 2006

2. Cataltepe O, Turanli G, Yalnizoglu D, Topçu M, Akalan N: Surgical management of temporal lobe tumor-related epilepsy in children. J Neurosurg 102 (3 Suppl):280-287, 2005

3. de Oliveira JG, Párraga RG, Chaddad-Neto F, Ribas GC, de Oliveira EP: Supracerebellar transtentorial approach-resection of the tentorium instead of an opening-to provide broad exposure of the mediobasal temporal lobe: anatomical aspects and surgical applications. Clinical article. J Neurosurg 116:764-772, 2012

4. Duvernoy HM: The Human Hippocampus: Functional Anatomy, Vascularization and Serial Sections With MRI, ed 3. Berlin: Springer-Verlag, 2005

5. Engel J Jr, Van Ness PC, Rasmussen T, Ojemann LM: Outcome with respect to epileptic seizures, in Engle J Jr (ed): Surgical Treatment of the Epilepsies, ed 2. New York: Raven Press, 1993, pp 609-621

6. Fernández-Miranda JC, de Oliveira E, Rubino PA, Wen HT, Rhoton AL Jr: Microvascular anatomy of the medial temporal region: part 1: its application to arteriovenous malformation surgery. Neurosurgery 67 (3 Suppl Operative):ons237ons 276,2010

7. Goel A, Shah A: Lateral supracerebellar transtentorial approach to a middle fossa epidermoid tumor. J Clin Neurosci 17:372-373, 2010

8. Izci Y, Seçkin H, Ateş O, Başkaya MK: Supracerebellar transtentorial transcollateral sulcus approach to the atrium of the lateral ventricle: microsurgical anatomy and surgical technique in cadaveric dissections. Surg Neurol 72:509-514, 2009

9. Jittapiromsak P, Deshmukh P, Nakaji P, Spetzler RF, Preul MC: Comparative analysis of posterior approaches to the medial temporal region: supracerebellar transtentorial versus occipital transtentorial. Neurosurgery 64 (3 Suppl):ons35ons43, 2009 
10. Kucukyuruk B, Richardson RM, Wen HT, Fernandez-Miranda JC, Rhoton AL Jr: Microsurgical anatomy of the temporal lobe and its implications on temporal lobe epilepsy surgery. Epilepsy Res Treat 2012:769825, 2012

11. Marcus H, Sarkar H, Mindermann T, Reisch R: Keyhole supracerebellar transtentorial transcollateral sulcus approach to the lateral ventricle. Neurosurgery 73 (2 Suppl Operative):E295-E301, 2013

12. Matjasko J, Petrozza P, Cohen M, Steinberg P: Anesthesia and surgery in the seated position: analysis of 554 cases. Neurosurgery 17:695-702, 1985

13. Moftakhar R, Izci Y, Başkaya MK: Microsurgical anatomy of the supracerebellar transtentorial approach to the posterior mediobasal temporal region: technical considerations with a case illustration. Neurosurgery 62 (3 Suppl 1):1-8, 2008

14. Nieuwenhuys R, Voogd J, van Huijzen C: The Human Central Nervous System, ed 4. Berlin: Springer-Verlag, 2008

15. Olivier A: Temporal resections in the surgical treatment of epilepsy. Epilepsy Res Suppl 5:175-188, 1992

16. Otani N, Fujioka M, Oracioglu B, Muroi C, Khan N, Roth P, et al: Thalamic cavernous angioma: paraculminar supracerebellar infratentorial transtentorial approach for the safe and complete surgical removal. Acta Neurochir Suppl 103:2936, 2008

17. Russell SM, Kelly PJ: Volumetric stereotaxy and the supratentorial occipitosubtemporal approach in the resection of posterior hippocampus and parahippocampal gyrus lesions. Neurosurgery 50:978-988, 2002

18. Ryvlin P, Kahane P: The hidden causes of surgery-resistant temporal lobe epilepsy: extratemporal or temporal plus? Curr Opin Neurol 18:125-127, 2005

19. Smith KA, Spetzler RF: Supratentorial-infraoccipital approach for posteromedial temporal lobe lesions. J Neurosurg 82:940-944, 1995

20. Tubbs RS, Oakes WJ: Relationships of the cisternal segment of the trochlear nerve. J Neurosurg 89:1015-1019, 1998

21. Türe U, Harput MV, Kaya AH, Baimedi P, Firat Z, Türe H, et al: The paramedian supracerebellar-transtentorial approach to the entire length of the mediobasal temporal region: an anatomical and clinical study. Laboratory investigation. J Neurosurg 116:773-791, 2012

22. Uchiyama N, Hasegawa M, Kita D, Yamashita J: Paramedian supracerebellar transtentorial approach for a medial tentorial meningioma with supratentorial extension: technical case report. Neurosurgery 49:1470-1474, 2001

23. Ueyama T, Al-Mefty O, Tamaki N: Bridging veins on the tentorial surface of the cerebellum: a microsurgical anatomic study and operative considerations. Neurosurgery 43:11371145,1998

24. Voigt K, Yaşargil MG: Cerebral cavernous haemangiomas or cavernomas. Incidence, pathology, localization, diagnosis, clinical features and treatment. Review of the literature and report of an unusual case. Neurochirurgia (Stuttg) 19:59 68,1976

25. Watanabe T, Katayama Y, Fukushima T, Kawamata T: Lateral supracerebellar transtentorial approach for petroclival meningiomas: operative technique and outcome. Clinical article. J Neurosurg 115:49-54, 2011

26. Weiner HL, Kelly PJ: A novel computer-assisted volumetric stereotactic approach for resecting tumors of the posterior parahippocampal gyrus. J Neurosurg 85:272-277, 1996

27. Wen HT, Rhoton AL Jr, de Oliveira E, Castro LH, Figueiredo EG, Teixeira MJ: Microsurgical anatomy of the temporal lobe: part 2-sylvian fissure region and its clinical application. Neurosurgery 65 (6 Suppl):1-36, 2009

28. Yonekawa Y, Imhof HG, Taub E, Curcic M, Kaku Y, Roth $\mathrm{P}$, et al: Supracerebellar transtentorial approach to posterior temporomedial structures. Technical note. J Neurosurg 94:339-345, 2001

\section{Author Contributions}

Conception and design: Bhatia, Weil. Acquisition of data: Weil, Middleton. Analysis and interpretation of data: Weil, Middleton, Niazi. Drafting the article: Bhatia, Weil, Middleton. Critically revising the article: all authors. Reviewed submitted version of manuscript: all authors. Study supervision: Bhatia, Niazi, Ragheb.

\section{Supplemental Information}

Previous Presentation

Partial results of this paper were presented in Toronto, Canada, at the Annual Meeting of the American Association of Neurological Surgeons, Section on Pediatric Neurological Surgery, December 3-6, 2013.

\section{Correspondence}

Sanjiv Bhatia, Division of Pediatric Neurosurgery, Miami Children's Hospital, Department of Neurosurgery, University of Miami/Miller School of Medicine, Miami, FL 33155. email: sanjiv.bhatia@mch.com. 